\title{
Sichern Sie sich den Zuschuss für Ihren neuen Kartenleser
}

\author{
Krankenkassen und Ärzte haben sich jetzt auf einen verbindlichen Fahrplan \\ für die Einführung der neuen Gesundheitskarten geeinigt. Praxisinhaber, die \\ sich den Zuschuss für das neue Lesegerät sichern wollen, haben dafür bis zum \\ September Zeit.
}

— Der Startschuss für die Ausgabe der neuen Gesundheitskarte soll bundesweit im Oktober dieses Jahres fallen. Den Krankenkassen werden 2\% ihrer Verwaltungskosten abgezogen, falls sie nicht bis Jahresende an mindestens 10\% ihrer Versicherten elektronische Gesundheitskarten ausgeben.

Nach der bereits Ende 2010 erfolgten Einigung auf die Höhe der Erstattungspauschalen (355 Euro für stationäre Lesegeräte, 280 Euro für mobile Leser und 215 Euro für die Installation) existiert auch ein Fahrplan, der besagt, dass die neuen Lesegeräte im zweiten und dritten Quartal auszuliefern seien.

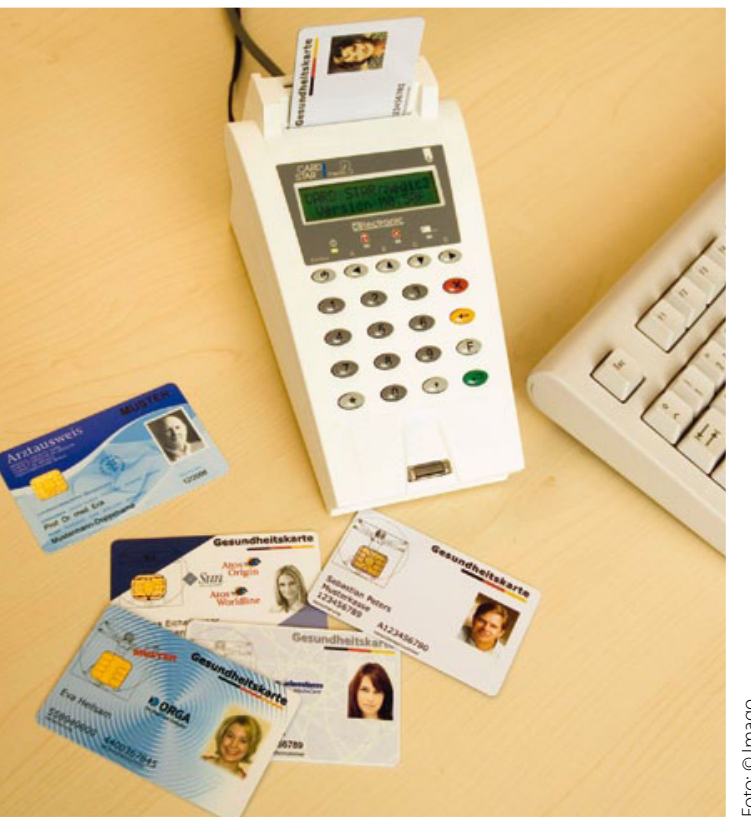

\section{Nicht auf den letzten Drücker bestellen}

„Lieferengpässe sind nicht zu befürchten“, so eine KBV-Sprecherin. Bei den Herstellern der Lesegeräte klingt das überwiegend ähnlich. Nur wenn die große Mehrheit der Kassenärzte erst auf den letzten Drücker, also im September, die Bestellungen für die Kartenleser aufgibt, könnte es am Ende eng werden, glauben Experten.

Die Vereinbarung lässt immerhin etwas Spielraum bei der Umsetzung in den Regionen: Die Gesamthöhe der Pauschale ist fix. Nicht endgültig fixiert sind der Ausgabezeitraum und auch die Berücksichtigung möglicher Praxisbesonderheiten.

Spannend wird auch die Inanspruchnahme der Pauschalen. Die Messlatte hat die KV Nordrhein gelegt. Dort riefen knapp 70\% der berechtigten Praxen die Pauschalen für die neuen eHealthBCS-Terminals beziehungsweise die mobilen Lesegeräte ab.

Beim GKV-Spitzenverband wird die Dauer des Gesamtrollouts auf „mindestens anderthalb Jahre" veranschlagt. Es liege dabei im Ermessen der einzelnen Krankenkasse, ob die alte KVK rasch ersetzt werde, oder ob das sukzessive erst beim Ablauf der Gültigkeit erfolge. Tatsache ist, dass KVK und eGK parallel gelten.

Für die eGK werden neue Lesegeräte benötigt.
In der Regel sollen die Krankenkassen die alten Karten einziehen, sobald eine neue Karte ausgegeben worden ist. Ärzte ohne eGK-Lesegerät werden also bei Patienten mit der neuen Karte buchstäblich schlechte Karten(leser) haben. Leistungen bei diesen Patienten abzurechnen könnte dann kompliziert und aufwendig werden.

Vorerst unterscheidet sich die eGK von der alten KVK nur im Lichtbild und in Angaben zum Geschlecht und zum Zuzahlungsstatus. Der Zuzahlungsstatus kann allerdings nicht aktualisiert werden. Die Aktualisierung des Zuzahlungsstatus (und anderer Stammdaten) erfordert eine Online-Anbindung. Wenn es dann soweit ist, werden Arztpraxen diese zur Verfügung stellen müssen. Nicht verpflichtet sind sie zur Ankopplung der Online-Verbindung an die Praxis-EDV.

\section{Erweiterte Funktionen nur mit Online-Anbindung}

Sollen allerdings Funktionen wie die bei der Bundesärztekammer angesiedelten elektronischen Notfalldaten genutzt werden, geht an der Praxis-EDV kein Weg vorbei. Die Notfalldaten liegen zwar auf der Karte, sie benötigen aber die Signaturfunktionen des elektronischen Arztausweises, die ohne Online-Anbindung nicht zu haben sind.

Vor Einführung der Onlinefunktionen steht eine Testphase, deren grober Rahmen vom Ministerium Mitte Januar festgezurrt wurde. Ende September muss gematik voraussichtlich - bisher ohne Fristsetzung - mindestens zwei Testregionen ausschreiben oder eine Testregion ausschreiben und eine selbst betreiben. 